\title{
Genus im DaF-Unterricht in Italien: Was machen die Lerner?
}

\author{
Marie Antoinette Rieger (Bologna)
}

\begin{abstract}
Gender classification in German is considered a rather intricate complex field of German grammar; gender selection is only partially rule-based and the existing rules are not only complex but their validity is often restricted as well. Therefore it is a received opinion that the acquisition of gender assignment is one of the most difficult problems learners of German as a foreign language have to face. Contrary to this general view, throughout many years of teaching German as a foreign language in Italy me and my colleague Marion Weerning (in this volume) did not receive the impression that gender really presents a major problem. Therefore we decided to carry out an empirical study. The design and the results of this study will be illustrated in the present paper. It will be shown that gender assignment is in fact more of a problem than was initially assumed. A detailed analysis demonstrates, however, that good and bad scores in gender assignment are not due to chance but can be explained by a combination of factors. The results also seem to indicate that learners develop a range of gender assignment rules in spite of the fact that gender is neither a frequent nor a systematically analysed topic in textbooks or in grammars.
\end{abstract}

\section{$1 \quad$ Einführung}

In der anhaltenden und intensiv geführten Auseinandersetzung um das Verhältnis von linguistischer und didaktischer Grammatik ${ }^{1}$ im Fremdsprachenunterricht wurden zwar "die Unterschiede zwischen wissenschaftlicher und pädagogischer Grammatik präzisiert und die Anforderungen [...] an didaktische Grammatiken/Lernergrammatiken herausgearbeitet" (Spillner 2003: 36), ${ }^{2}$ aber die praktischen Ergebnisse sind noch nicht in jedem Fall wirklich zufriedenstellend. ${ }^{3}$ Wie Marion Weerning (in diesem Band) zeigt, ist ein Bereich des Unterrichts Deutsch als Fremdsprache (DaF), in dem sich das Verhältnis zwischen Systemwissen und Unterricht als besonders schwierig erweist, das Genus. Befragt man linguistische Grammatiken, so zeigt sich, dass das deutsche Genussystem durch eine außerordentliche Komplexität gekennzeichnet ist, denn in kaum einem anderen Bereich "sind so viele formale und semantische Regeln zur halbwegs erschöpfenden Beschreibung des Systems erforderlich" (Krohn/Krohn 2008: 10). In vielen Lehr- und Lernmaterialien für DaF wird die Genuszuweisung immer noch als regellos dargestellt (vgl. Weerning in diesem Band), und dort, wo die Systemhaftigkeit erkannt wird, gilt sie als nicht lehrbar. So heißt es beispielsweise in der Grammatik von Helbig/Buscha (2001: 245):

Das beherrschende Genussystem im Deutschen ist ein formalgrammatisches System, das in seinen Systemzügen nur im Rahmen einer historischen Grammatik erklärt und übersichtlich gemacht werden kann.

1 Didaktische Grammatik steht hier und im Folgenden als Oberbegriff und schließt sowohl lehrwerkunabhängige als auch lehrwerkbezogene Lehrer- und Lernergrammatiken ein.

2 Einen kurz gefassten Überblick zum Themenkomplex mit weiteren Literaturhinweisen gibt Zimmermann (2003).

3 Neben einigen kritischen Hinweisen in Zimmermann (2003) vgl. hierzu insbesondere Ivancic (2003). 
Dieser Aussage folgen seitenlange Aufzählungen von semantischen und formalen Regeln, die in dieser Form tatsächlich kaum "praktikable Regeln" (ebd.) abgeben. Hier und anderswo werden Lehrende mit der "Lehrstrategie", zu jedem Substantiv einfach den genusanzeigenden bestimmten Artikel mitlernen zu lassen, allein gelassen, und Lernende durch den ungeheuren mentalen Aufwand, der bei Einsatz der entsprechenden "Lernstrategie" nötig wäre, vor eine geradezu unlösbare Aufgabe gestellt. Im Bereich Genus kommen didaktische Grammatiken ihrem originären Auftrag, nämlich "durch kluge didaktische Konzepte das Fremdsprachenlernen zu erleichtern" (Zimmermann 2003: 408), also nur sehr unzureichend nach. Umso erstaunlicher ist der Mangel an gut durchdachten didaktischen Aufbereitungen, wenn man bedenkt, dass der Genuserwerb gleichzeitig als eines der größten Lernprobleme gilt: "Gender assignment in German is not considered a very transparent field of German grammar; its acquisition presents one of the most difficult problems for adult learners of German" (Wegener 2000: 511).

Allerdings gibt es auch Studien, die diese Sicht relativieren. So kann Christen (2000: 175) in ihrer Untersuchung von DaF-Schülerarbeiten aus der 4. bis 12. Klasse "einen beträchtlichen Anteil von richtigen Genuszuweisungen" nachweisen. Dass dabei keine schulstufen- bzw. altersspezifischen Unterschiede festzustellen sind, hatte sie durchaus erwartet, denn der Genuserwerb weist im Vergleich zu anderen Bereichen des Spracherwerbs eine Besonderheit auf:

Die aufgeführten Werte machen deutlich, dass bei der Genuszuweisung nicht mit einer kontinuierlichen Zunahme richtiger Genera gerechnet werden kann, weder auf individueller Ebene, noch über die Schulstufen hinweg. Dieser Sachverhalt erstaunt kaum, weil mit der kontinuierlichen Zunahme des Wortschatzes gleichzeitig bei immer wieder neuen Nomen auch das zugehörige Genus selektioniert werden muss. Es handelt sich ja nicht um die lernersprachliche Fähigkeit, bei einem ganz bestimmten Nomen "immer besser" zu wissen, welches das jeweilige Genus ist. (ebd.; Hervorhebung im Original)

Die Ergebnisse von Christen unterstützen die von Marion Weerning und mir im Laufe von vielen Jahren DaF-Unterricht an italienischen Goethe-Instituten ${ }^{4}$ entwickelte, aber bisher nicht weiter reflektierte Überzeugung, nämlich dass Genus kein herausragendes Lernproblem ${ }^{5}$ darstellt. Bei genauerer Betrachtung könnte sich dieser Eindruck zum einen aber auch damit erklären, dass Genusfehler oft nicht als solche erkannt, sondern als Kasusfehler "abgetan" werden. So ist durchaus denkbar, dass bei einem Ausdruck wie es dauert ein Monat eher die fehlende maskuline Akkusativendung als die Zuweisung des Neutrums zu Monat wahrgenommen wird. Im Kontext $\mathrm{DaF}$ in Italien ist dies durchaus plausibel, da das entsprechende mese 'Monat' und alle Monatsnamen wie im Deutschen maskulin sind, das Genus also als mögliche Fehlerquelle nicht sofort in den Vordergrund tritt. Unterschiede zwischen den beiden Sprachen ergeben sich dagegen daraus, dass bei der italienischen Entsprechung dura un mese der Akkusativ sich nicht vom Nominativ unterscheidet. ${ }^{6}$ Zum anderen behindert falscher Genusgebrauch die Kommunikation in der Regel nicht, wodurch er in einem stark kommunikativ ausgerichteten und insbesondere an pragmatischer Adäquatheit interessierten Unterricht also mehr oder weniger "überhört" wird. Dass erfolgreiche Kommunikation auch ohne ein voll entwickeltes Genussystem möglich ist, bestätigt Wegener (2000: 536) auch in Bezug auf den Zweitspracherwerb von Kindern:

4 In dieser Zeit, d. h. von 1985-1999 ca. wurden unsere Kurse überwiegend von jungen Erwachsenen besucht, gehörten also derselben Altersgruppe an wie die Versuchspersonen der hier vorliegenden Untersuchung und sind damit auch mit den Zwölftklässlern der Studie von Christen durchaus zu vergleichen.

5 Die Betonung liegt hier auf herausragend. Natürlich machten und machen auch unsere LernerInnen (zunächst KursteilnehmerInnen der italienischen Goethe-Institute Genua und Palermo, heute Studierende der Universitäten Bologna und Palermo) Genusfehler.

6 Mir ist durchaus bewusst, dass es sich hier um nicht belegtes Erfahrungswissen handelt. Auch geht es an dieser Stelle nicht darum, ob die Fehleranalyse seitens der Lehrperson den tatsächlichen Fehlerursachen bei einem bestimmten Lernenden in einer bestimmten Situation entspricht. 
The gender acquisition of the children under investigation also shows that interactionistic explanations of language acquisition are not sufficient. The advanced among the Turkish children demonstrate the astonishing phenomenon that syntactic abilities can be developed all the way to the formation of complex sentences without the parallel development of inflectional morphology. As a result, smooth communication in everyday interaction is possible without complying with gender.

So wollten wir empirisch überprüfen, ob die Unauffälligkeit von Genusfehlern in unserem Unterrichtsalltag eventuell auf Faktoren wie mangelnde Aufmerksamkeit seitens der Lehrenden zurückzuführen ist oder ob die Genuszuweisung - zumindest für unsere italienischen Deutschlernenden - tatsächlich kein herausragendes Lernproblem darstellt. ${ }^{7}$

Die vorliegende Arbeit gliedert sich in 5 Abschnitte. Im Anschluss an diesen in die Fragestellung einführenden Teil wird in Abschnitt 2 dargestellt, welche Eigenschaften des deutschen Genussystems für Lernschwierigkeiten verantwortlich gemacht werden können. Abschnitt 3 erläutert das Forschungsdesign im Hinblick auf die gewählte Erhebungsmethode, die Auswahl und Anordnung der Items sowie die Versuchspersonen (VP). Dargestellt und interpretiert werden die Ergebnisse in Abschnitt 4. Abschnitt 5 beschließt den Beitrag mit einigen didaktischen Schlussfolgerungen, die aus den Ergebnissen der Studie gezogen werden können.

\section{Genus als Lernproblem aus systemlinguistischer Sicht}

Eine heute weithin anerkannte Definition der Kategorie Genus geht auf Hockett (1958: 231, zitiert z. B. in Corbett 1991: 1; Unterbeck 2000: XV) zurück: "Genders are classes of nouns reflected in the behavior of associated words". Einer Sprache wird also dann ein Genussystem zugesprochen, wenn zwischen Substantiv und den ihm untergeordneten Elementen innerhalb oder außerhalb der Nominalphrase Kongruenzphänomene zu beobachten sind. Als bis heute ungeklärt gelten muss allerdings die Frage nach der Funktion, die eine nominale Klassenbildung - und damit letztendlich die Kategorie "Genus" - überhaupt hat: "Genus gehört zu den Kategorien, deren grammatische Bedeutung erst noch entdeckt werden muß" (Leiss 1994: 290), eine Aussage, die wiederholt auch in jüngeren Veröffentlichungen immer noch zu finden ist, so z. B. bei Bittner (2000: 1):

[...] let it be remarked that at present there is probably no one who can say what gender as a grammatical category really is. It is unclear what grammatical function is associated with the division of nouns into two, three, or more classes. All investigations to date that have dealt with grammatical phenomena related to gender [...] could not answer the question of which grammatical relations and/or functions would not be expressible if the nouns were not separated into different classes.

Allgemeiner Konsens besteht heute darin, dass sich (in den indo-europäischen Sprachen) das grammatische Genus nicht aus dem natürlichem Geschlecht durch Übertragung vermeintlich männlicher bzw. weiblicher Eigenschaften entwickelt hat, wie das so vehement von Jacob Grimm und seinen Anhängern vertreten wurde. ${ }^{8}$ Favorisiert wird dagegen der umgekehrte Prozess, nämlich die Entstehung von Sexus als Sekundärableitung aus dem Genus, wobei es heute eine Reihe von Arbeiten gibt (vgl. Leiss 1994 und 2000; Vogel 2000; Weber 2000), die die ursprüngliche Funktion der Genusmarkierung in einer Art von Nominalaspekt sehen zum

\footnotetext{
7 An dieser Stelle möchte ich ausdrücklich darauf hinweisen, dass es hier nicht darum geht, Hypothesen über die Art des Erwerbs des Genussystems zu testen. Die im Folgenden beschriebene Untersuchung möchte vielmehr Erfahrungen und Wahrnehmungen aus der Praxis auf den Prüfstand stellen. Ausführlich diskutiert wird der erwerbstheoretische Hintergrund in Bezug auf DaF-LernerInnen sowohl im Hinblick auf den Forschungsstand als auch auf die aus den jeweiligen Studien zu ziehenden Schlussfolgerungen in den Arbeiten von Diehl et al. (2000), Menzel (2004), Thomoglou (2004) und Krohn/Krohn (2008).

8 Ausführlich beschrieben und dokumentiert wird dieser Streit zwischen romantisierender Metaphorik und nüchternem Formalismus in Sieburg (1997).
} 
Ausdruck von Definitheit vs. Indefinitheit bzw. zur Unterscheidung von Individual- und Kollektivnomen:

It is proposed that the original function of noun classification in general, and gender in particular, was a differentiation of noun concepts with regard to quantity, i. e., individuality and continuativity. Concerning gender, this was represented by the categories masculine vs. feminine/neuter. This differentiation is still valid and is motivated in the secondary vocabulary within the realm of nominal abstracts. (Vogel 2000: 489)

Allen - mehr oder minder plausiblen - Erklärungsversuchen ist gemeinsam, dass die wie auch immer geartete ursprüngliche Bedeutung verblasst ist. Diese offenbare Funktionslosigkeit wird als ein Grund angesehen, warum Genus im Zweit- und Fremdsprachenerwerb ein Lernproblem darstellt. ${ }^{9}$ So konstatiert Wegener (1995: 7) in ihrer Untersuchung zum Zweitspracherwerb von Schulkindern:

Die Erwerbsdaten zeigen, daß für den Lerner die Markierung von Kasus und/oder Numerus Vorrang hat, da diese Kategorien funktional sind, daß das fast völlig funktionslose Genus dagegen für ihn in den Hintergrund tritt.

Neben der - auch anderswo - nicht eindeutig beantworteten Frage nach der Funktion, stellt das deutsche Genussystem DaF-Lernende vor zwei weitere grundsätzliche Probleme. Wie bereits erwähnt, ist zum einen die Genuszuordnung, also die Aufteilung der Nomen auf die drei Genusklassen, sehr komplex und erfolgt aus heutiger Sicht nur zum Teil regelgeleitet. Zum anderen ist die richtige Genuszuweisung durch die Lernenden "bloss ein erster 'Teilerfolg', der noch keineswegs die zielsprachlichen Flexive aller genussensitiven Elemente und damit formale Korrektheit impliziert" (Christen 2000: 169; Hervorhebung im Original). Was den ersten Punkt betrifft, so kann laut der Untersuchungen von Wegener (1995: 2f.) im deutschen Grundwortschatz das Genus in knapp 60\% der Fälle aufgrund formaler Kriterien zugewiesen werden, und zwar aufgrund von vier phonologischen bzw. morphologischen Grundregeln: Regel 1 ist als "Schwa-Regel" bekannt und besagt, dass Substantive auf - $e$ zu etwa 90\% feminin sind; Regel 2 besagt, dass Substantive auf -el, -en und -er zu etwa 65\% maskulin sind; Regel 3 ist als "Einsilberregel" bekannt und besagt, dass die meist einsilbigen Kernwörter des deutschen Wortschatzes zu etwa 50\% maskulin sind. Regel 4 bezieht sich auf die Tatsache, dass Ableitungssuffixe das Genus des Substantivs bestimmen. Außerdem kann in weiteren knapp 6\% der Fälle das Genus aufgrund der Regel "Bezeichnungen für männliche Lebewesen sind im unmarkierten Fall Maskulina, solche für weibliche Feminina" (Wegener 1995: 2) abgeleitet werden. Nimmt man alle Fälle zusammen, so erfolgt etwa zwei Drittel der Genuszuweisungen im Grundwortschatz regelgeleitet.

Das Lernproblem ergibt sich aber nicht nur deshalb, weil bei etwa einem Drittel des nominalen Grundwortschatzes für die Genuszuweisung keine Regeln zur Verfügung stehen, sondern auch, weil einzelne Regeln nur eine begrenzte Validität besitzen. Für Lernende ist so z. B. nicht ersichtlich, warum die Regel "Substantive auf -el sind oft maskulin" (nach Wegener 1995: 3 in 60,5\% der Fälle) bei Schlüssel und Apfel greift, nicht aber bei Schüssel und Ampel. Damit liegt das Deutsche wohl irgendwo in der Mitte des Kontinuums von overt und covert gender:

Languages in which the gender of a noun is evident from its form are often described as having 'overt gender'; those where gender is not shown by the form have 'covert' gender. [...] However, the distinction is much less rigid than is often implied. There are many possibilities between the poles of absolutely overt and absolutely covert. (Corbett 1991: 62)

\footnotetext{
9 Zum Genuserwerb im frühen bilingualen Spracherwerb vgl. z. B. Müller (2000); zum Genuserwerb in der
} Erstsprache findet sich ein Überblick in Krohn/Krohn (2008: 80-86). 
Aber nicht nur die insgesamt eher begrenzte Durchsichtigkeit des Genussystems macht den Bereich für Lernende problematisch. Denn die Kategorie Genus beinhaltet ja - und damit komme ich zur zweiten oben genannten Erwerbshürde - auch bestimmte Kongruenzphänomene. Im Deutschen ergibt sich dabei eine spezielle Problematik daraus, dass die Marker an den mit dem Substantiv in Verbindung stehenden Elementen erstens nicht nur Genus, sondern auch Numerus und Kasus anzeigen und zweitens weniger Marker als zu unterscheidende Kategorien zur Verfügung stehen, die Marker also polyvalent sind:

Das Genus besteht aus einem ganzen Flexionsparadigma, dessen einzelne Formen sich zusätzlich nach Kasus und Numerus ausrichten. Die einzelnen Flexive sind aber nicht eineindeutig, sondern mit bloss 8 verschiedenen Suffixen (nämlich $-\varnothing,-e,-e r,-e n,-e m,-e s,-a s,-i e$ ) werden alle möglichen Genus-Kasus-Numerus-Kombinationen ausgedrückt. [...] Für Lernende ergibt sich aber aus der deutschen Formenökonomie rsp. der deraus [sic] resultierenden Polysemie einerseits ein erhebliches Analyseproblem beim Input [...], andererseits ein Syntheseproblem beim Output [...]. (Christen 2000: 168f.) ${ }^{10}$

\section{$3 \quad$ Das Untersuchungsdesign 11}

Die empirische Untersuchung sollte insbesondere die beiden folgenden Hypothesen testen:

1. Das Genus wird im Bereich des Grundwortschatzes auch ohne (systematische) Unterweisung in einem zufriedenstellenden Ausmaß erworben.

2. Die Lernenden verfügen in Bezug auf die Genuszuweisung über ein geringes bewusstes und/oder verbalisierbares Regelwissen.

Mit Hypothese 1 stellen wir nicht nur die verbreitete Überzeugung, Genus sei ein herausragendes Lernproblem, in Frage, sondern widersprechen auch Thomoglou (2004: 65), die - sehr kategorisch - meint: "Die Schwierigkeiten, die die Fremdsprachlerner beim Genuserwerb haben, sind auf die Systematik der Lehrwerke zurückzuführen". Hypothese 2 kann als vorsichtige erwerbstheoretische Annahme interpretiert werden, nämlich dass zwischen dem zufriedenstellenden Meistern der Lernaufgabe "Genus" und dem bewussten Regelwissen kein starker Zusammenhang besteht.

Solch verallgemeinernde Aussagen müssen an einer möglichst großen Versuchsgruppe auf der Grundlage möglichst vieler Items und Regeln getestet werden. Nachdem sich die Auswertung von Prüfungsarbeiten als undurchführbar erwiesen hatte (vgl. Fußnote 10), wählten wir als Erhebungsmethode den Fragebogen. Um die Bereitschaft zum Ausfüllen und die Konzentrationsfähigkeit der VP nicht über Gebühr zu strapazieren, sollte die Bearbeitungszeit $30 \mathrm{Mi}-$ nuten nicht überschreiten. Zur Erhöhung der Validität sollten außerdem die Items mehrfach abgefragt, jede Regel durch mehrere Items vertreten und verschiedene Testformate eingesetzt werden. Aus diesen Vorgaben ergibt sich eine Höchstzahl von 50 Items. In allen drei Testformaten (s. u.) steht jedes Item im Nominativ Singular und das Genus soll in Form des be-

\footnotetext{
${ }^{10}$ Die Uneindeutigkeit der Marker hat auch dazu geführt, dass wir unser ursprüngliches Vorhaben, Genusdaten nicht isoliert, sondern aus bereits vorhandenen studentischen Arbeiten zu erheben, schließlich aufgegeben haben. So kann beispielsweise bei Ich habe ein Wagen nicht zweifelsfrei entschieden werden, ob es sich bei ein Wagen um eine falsche Kasuszuweisung (Nominativ statt Akkusativ) oder eine falsche Genuszuweisung (Neutrum statt Maskulinum) handelt. Die Zahl solcher und ähnlicher Zweifelsfälle übersteigt bei weitem die der eindeutigen. Außerdem ist uns während der Auswertung der Texte (B1-Niveau) bewusst geworden, wie selten die Lernenden von der pronominalen Wiederaufnahme - die ja ein hilfreiches Indiz wäre - Gebrauch machen. Und selbst aus korrekten Ausdrücken wie im Jahr kann nicht eindeutig abgeleitet werden, dass tatsächlich das Neutrum zugewiesen wurde. Eine ausführliche Diskussion der Problematik der Erhebungsmethode findet sich z. B. in Krohn/Krohn (2008: 16-18).

${ }^{11}$ Regelauswahl, Zusammenstellung der Items und Strukturierung des Fragebogens sind eine Gemeinschaftsarbeit von Marion Weerning und mir.
} 
stimmten Artikels der, die, das zugewiesen werden. Alle Fragen und Aufgabenstellungen sind auf Italienisch formuliert, um sprachlich bedingte Verständnisprobleme möglichst auszuschließen.

Die Auswahl der Items ist ein Zusammenspiel von Lernniveau und Zuweisungsregeln. Jede Regel ist durch drei valide, also der Regel entsprechende, und - wo möglich ${ }^{12}$ - zwei nichtvalide, also der Regel nicht entsprechende Items vertreten. Zwei valide Items und ein nichtvalides Item stammen aus dem Grundwortschatz (GWS), je ein valides und ein nicht-valides Item entsprechen einem Niveau oberhalb von B2 (B2+). ${ }^{13}$ Aus dem GWS werden Items gewählt, die spätestens auf A2-Niveau produktiv bekannt sein sollten, wo dies nicht möglich ist, auf B1-Niveau. Für die Regel "Substantive auf $-e$ sind meist feminin" ("Schwa-Regel") z. B. sind dies Reise, Blume und Junge. Da die VP mindestens B1-Niveau erreicht hatten, erwarteten wir im Sinne von Hypothese 1 für alle, auch für die nicht-validen Items aus dem GWS eine hohe Trefferquote. Das nicht-valide Item des GWS ist, wo immer möglich, so gewählt, dass die richtige Genuszuweisung aufgrund einer anderen Regel möglich ist. So kann für Junge, wenn man die Bedeutung kennt, das richtige Genus aufgrund des natürlichen Geschlechts zugewiesen werden. Allerdings kann von einer richtigen Genuszuweisung natürlich nicht geschlossen werden, ob eine Regel angewendet wurde, das Genus Teil des mentalen Lexikoneintrags ist oder die richtige Zuweisung einfach erraten wurde. Die beiden Items B2+ sind für die Gruppe der "Schwa-Regel" Waise und Funke. Im Sinne von Hypothese 2 erwarten wir für diese den Lernenden im Allgemeinen unbekannten Items eine im Zufälligkeitsbereich liegende Trefferquote. Eine über dem Zufall liegende Trefferquote würde bei dem validen Beispiel dagegen auf die Anwendung von Regelwissen hindeuten. Dasselbe gilt, wenn Funke in relevantem Ausmaß feminines Genus zugewiesen wird. Soweit möglich haben die gewählten Items ein anderes Genus als das italienische Äquivalent, um bei richtiger Genuszuweisung muttersprachlichen Transfer ausschließen zu können. Die insgesamt 49 Items setzen sich aus 19 Maskulina, 16 Feminina und 14 Neutra zusammen.

Unter diesen Vorgaben ist es möglich, 11 Regeln abzudecken. Auch die Auswahl der Regeln folgt mehreren Kriterien. Zum einen sollte es den Verhältnissen des Deutschen entsprechend semantische, morphologische und phonologische Regeln geben. Zum anderen sollten aus dem Unterricht wahrscheinlich bekannte Regeln, aber auch solche abgedeckt werden, die im DaFUnterricht erfahrungsgemäß keine Rolle spielen (vgl. Weerning in diesem Band). ${ }^{14}$ Die 11 Regeln sind in ihrer Kurzform: ${ }^{15}$ 1. Bei unmarkierten Personenbezeichnungen entspricht das grammatische Genus dem natürlichen Geschlecht (86,1\%); 2. Alkoholische Getränke sind maskulin mit Ausnahme von Bier; aus Sicht der Lernenden können Regel 1 und 2 nur angewendet werden, wenn die Bedeutung des Substantivs bekannt ist. 3. Substantive auf -ung sind feminin (100\%); 4. Substantivierte Verben sind neutrum (100\%); damit diese Regel angewendet werden kann, muss erkannt werden, dass eine verbale Basis zugrunde liegt. 5. Movie-

\footnotetext{
${ }^{12}$ Einige der von uns gewählten Regeln haben eine Validität von $100 \%$, so dass sich keine Ausnahmen finden lassen. Regeln mit einer Validität von weniger als $100 \%$ werden also durch insgesamt 5 Items (3 Grundwortschatz +2 B2-Niveau und höher, dabei für jede der beiden Niveaugruppen je ein nicht valides), Regeln mit absoluter Validität durch 3 Items (2 Grundwortschatz + 1 B2-Niveau und höher) vertreten. Die Einschränkung wo möglich ist im Folgenden immer mitzudenken.

13 Der Auswahl liegt der in Profile deutsch (Glaboniat et al. 2002) nach Niveaustufen geordnete Wortschatz zugrunde.

14 Vgl. auch Christen, die darauf hinweist, dass in von Genfer Lehrenden selbst entwickelten Materialien Genusregeln formuliert werden und zwar die Regel des natürlichen Geschlechts sowie Regeln in Verbindung mit Wortbildungssuffixen (2000: 172, Fußnote 64)

15 Die nach den Regeln geordnete Liste aller Items findet sich im Anhang. Die folgenden Validitätsangaben stammen aus Wegener (2000: 513). Der Skopus wird hier nicht berücksichtigt und die Auszählung verschiedener Wortschätze kann durchaus zu unterschiedlichen Angaben führen. Trotzdem erhält man durch die Angaben zumindest eine grobe Vorstellung davon, in welchem Validitätsbereich sich jede Regel bewegt.
} 
rungen auf -in sind feminin $(100 \%) ; 16$ 6. Substantive auf $-e$ sind fast immer feminin $(90,5 \%)$; 7. Bezeichnungen von Personen und Geräten aus deverbaler Derivation auf -er sind meist maskulin; wie bei Regel 1 und 2 kann die morphologische Regel nur angewendet werden, wenn die Bedeutung des Substantivs bekannt ist. Allerdings handelt es sich hierbei auch um eine phonologische Regel, die - ohne Rücksicht auf zugrunde liegende Wortbildungsmuster besagt, dass nahezu 65\% der Substantive auf -er maskulin sind. 8. Durch deverbale und denominale Derivation gebildete Substantive auf - $e l$ sind meist maskulin; als phonologische Regel sind Substantive auf - el zu 60,5\% maskulinum. 9. Substantive aus denominaler Derivation mit $G e-. . .(-e)$ zur Bildung von Kollektiva sind neutrum; auch hier muss für eine sichere Anwendung der Regel die Bedeutung des Substantivs bekannt sein. 10. Substantive auf -en wenn keine substantivierten Verben - sind überwiegend maskulinum $(72,1 \%)$; 11. einsilbige Substantive mit Konsonanten im An- oder Auslaut sind oft maskulin $(51,8 \%) .{ }^{17}$

Damit gibt es fünf Regeln für das Maskulinum (m), 3 für das Femininum (f) und 2 für das Neutrum (n) sowie die Regel "Genus ist Sexus", die sowohl für das Maskulinum als auch das Femininum gelten kann. Für die semantische Regel haben wir uns deshalb für die Gruppe der Alkoholika - und damit nur indirekt für Maskulinum - entschieden, weil bei anderen (als bekannt vorausgesetzten) semantischen Regeln Übereinstimmung mit dem Italienischen besteht und/oder alle Items aus dem GWS stammen. So besteht bei den morpho-phonologischen Regeln ein Verhältnis von 4 m, $3 \mathrm{f}$ und $2 \mathrm{n}$. Allerdings sind die Regeln, wie der obige Überblick zeigt, von sehr unterschiedlicher Qualität. Dabei heben sich insbesondere die Regeln für Feminina $\mathrm{ab}$, denn sie erreichen nicht nur eine nahezu absolute Validität, sondern die entsprechenden Substantive sind auch auffällig morphologisch markiert. Die Regeln für Maskulina und Neutra haben dagegen eine viel geringere Validität und/oder tragen keine auffälligen morphologischen Kennzeichen.

Der Fragebogen besteht aus zwei Teilen, wobei im ersten Teil biographische Daten erhoben werden wie Länge des Deutschlernens, Besitz eines Sprachdiploms, außerunterrichtliche Kontakte mit Deutschsprachigen u. a.m. Der das Genus betreffende zweite Teil sieht drei verschiedene Aufgaben vor. In Aufgabe 1 werden alle 49 Items in einer nach dem Zufallsprinzip angeordneten Liste präsentiert und die VP müssen nur das Genus bestimmen. In Aufgabe 2 wird von jeder der 11 Regeln ein valides Item präsentiert und zwar abwechselnd aus dem GWS und aus B2+. Die VP müssen wieder das Genus in Form des bestimmten Artikels notieren, aber bei jedem Item auch frei formulieren, aufgrund welcher "Überlegungen" das Genus bestimmt wurde. In der Aufgabenstellung wird der Begriff Regel bewusst vermieden, um die VP möglichst wenig zu beeinflussen. In Aufgabe 3 schließlich werden 19 weitere Items (aus der Liste) zusammen mit 7 möglichen Zuweisungskriterien präsentiert. Jetzt sollen die VP neben dem Genus auch angeben, nach welcher der vorgegebenen "Überlegungen" der Artikel gewählt wurde. Die zur Auswahl stehenden Kriterien sehen einige sehr allgemein formulierte Regeln vor (semantische Regel des natürlichen Geschlechts sowie in Bezug auf bestimmte Sachgruppen; morphologische Regel in Bezug auf Suffixe), aber auch Optionen wie Artikel zusammen mit dem Substantiv gelernt; Artikel klingt gut; derselbe Artikel wie im Italienischen. Schließlich gibt es noch Sonstiges. ${ }^{18} \mathrm{Da}$ aus den oben erwähnten Durchführbarkeitsüberlegungen in Aufgabe 2 und 3 nicht alle Items abgefragt werden können, gibt es eine A- und eine B-Version des Fragebogens. In Aufgabe 1 sind in beiden Versionen alle Items

\footnotetext{
16 Regel 4 und 5 wird bei Wegener (2000: 513) nicht berücksichtigt.

${ }^{17}$ Die von Köpcke/Zubin $(1983,1984,1996)$ aufgestellten phonetischen Einsilber-Regeln sind in ihrer Komplexität für den DaF-Unterricht nicht praktikabel. Allerdings scheinen prototypische Maskulina im GWS einsilbig zu sein mit Konsonanten im Wortan- und -auslaut (vgl. Thomoglou 2004: 43). Dies wird hier mit Regel 11 intendiert.

18 Die Antworten zu Aufgabe 2 und 3 konnten innerhalb des vorliegenden Zeitrahmens nicht detailliert ausgewertet werden. Einige allgemeine Ergebnisse finden sich gegen Ende von Abschnitt 4.
} 
aufgelistet, allerdings je unterschiedlich angeordnet, um Reiheneffekte ausschließen zu können. In Aufgabe 2 und Aufgabe 3 sind die Items gleichmäßig auf die beiden Fragen und die beiden Fragebogenversionen verteilt, so dass - außer dem zweiten validen Item des GWS zwar alle Items einmal vorkommen, aber jede VP in Aufgabe 2 und 3 zusammengenommen nur 30 statt 49 Items bearbeitet.

Die hier ausgewertete Befragung wurde an der Sprach- und Literaturwissenschaftlichen Fakultät der Universität Bologna durchgeführt und zwar in allen 3 Jahren des BA-Studiengangs, in dem die Studierenden Deutsch in der Regel als 1. oder 2. Fremdsprache studieren. Die Studierenden wurden während der jeweiligen Grundkurse zur deutschen Sprachwissenschaft gebeten, den Fragebogen auszufüllen. ${ }^{19}$ Dies nicht nur, um einen möglichst hohen Rücklauf zu garantieren. Um sicherzustellen, dass die Studierenden auf ihr eigenes Wissen zurückgreifen, musste auch die Befragung externer Quellen wie Wörterbücher usw. ausgeschlossen werden. Verhindert werden sollte aber auch ein Abgleichen der Antworten in den verschiedenen Aufgabenstellungen. Deshalb wurde zum einen für jede einzelne Aufgabe eine relativ knappe Zeitspanne vorgegeben und den VP wurde das Hin- und Herblättern untersagt. Von insgesamt 101 ausgefüllten Fragebogen wurden 97 ausgewertet, da es sich bei 3 VP um deutsche MuttersprachlerInnen handelt und eine VP der Aufgabe aufgrund sprachlicher Defizite nicht gewachsen war. Von diesen 97 waren 48 aus dem 1. Studienjahr, allerdings mit guten Vorkenntnissen (im Allgemeinen B1, mindestens aber A2, da der Kurs auf Deutsch gehalten wurde), 35 aus dem 2 . und 14 aus dem 3 . Studienjahr. ${ }^{20}$

\section{$4 \quad$ Ergebnisse}

Für eine Bestätigung von Hypothese 1 - DaF-Lernende erwerben Genus im Bereich des GWS in einem zufriedenstellenden Ausmaß - muss bei den Items aus dem GWS die Trefferquote in einem ausreichend hohen Bereich liegen. In Anlehnung an die Überlegungen von Krohn/Krohn (2008: 124-126) soll eine Trefferquote von mindestens 75\% bedeuten, dass das Genus kein herausragendes Lernproblem darstellt. Die folgende Tabelle stellt die Trefferquoten im Überblick dar:

${ }^{19}$ An dieser Stelle möchte ich meinen beiden Kolleginnen Eva-Maria Thüne und Barbara Ivancic danken, die einen Teil ihrer Unterrichtszeit für die Befragung zur Verfügung gestellt haben.

${ }^{20}$ Bisher konnten die Fragebogen noch nicht studienjahrbezogen ausgewertet werden. Allerdings ist beim Erfassen der Daten stark der Eindruck entstanden, dass die Gruppe aus dem ersten Studienjahr, frisch von der Schule und viele mit dem Zertifikat Deutsch, besser abschneidet als die anderen beiden Gruppen. Dies steht im Einklang mit der Beobachtung von Christen, dass bei der Genuszuweisung keine kontinuierliche Verbesserung zu verzeichnen ist. 


\begin{tabular}{|c|c|c|c|c|c|c|}
\hline & \multicolumn{2}{|c|}{ alle Items } & \multicolumn{2}{|c|}{ GWS } & \multicolumn{2}{|c|}{ B2+ } \\
\hline & $\mathbf{n}$ & $\%$ & $\mathbf{n}$ & $\%$ & $\mathbf{n}$ & $\%$ \\
\hline gesamt & 49 & 58,35 & 30 & 66,01 & 19 & 46,25 \\
\hline $\mathbf{m}$ & 19 & 58,15 & 12 & 65,95 & 7 & 44,77 \\
\hline f & 16 & 69,29 & 11 & 67,59 & 5 & 73,02 \\
\hline $\mathbf{n}$ & 14 & 46,11 & 7 & 63,61 & 7 & 28,61 \\
\hline valide Items & 33 & 67,23 & 22 & 72,35 & 11 & 56,99 \\
\hline $\mathbf{m}$ & 17 & 59,46 & 11 & 64,77 & 6 & 49,72 \\
\hline $\mathbf{f}$ & 10 & 89,56 & 7 & 92,82 & 3 & 81,96 \\
\hline $\mathbf{n}$ & 6 & 52,04 & 4 & 57,39 & 2 & 41,34 \\
\hline nicht valide Items & 16 & 40,02 & 8 & 48,56 & 8 & 31,48 \\
\hline $\mathbf{m}$ & 2 & 47 & 1 & 78,94 & 1 & 15,06 \\
\hline f & 6 & 35,5 & 4 & 23,45 & 2 & 59,62 \\
\hline n & 8 & 41,66 & 3 & 71,91 & 5 & 23,52 \\
\hline
\end{tabular}

Tab. 1: Trefferquoten im Überblick ${ }^{21}$

Die Auswertung ergibt für alle 97 ausgewerteten Fragebogen, alle 49 Items und alle Aufgaben eine durchschnittliche Korrektheitsquote von 58,35\%. Im Bereich des GWS (30 Items) liegt die Trefferquote bei 66,01\% und damit (weit) unterhalb unserer Erwartungen. Für die 19 Items im Bereich B2+ werden immerhin 46,25\% erreicht. Auch wenn Erhebungsmethode, Probanden und Item-Auswahl sehr unterschiedlich sind, liegen unsere Ergebnisse doch im Trend anderer neuerer Untersuchungen: die Bologneser VP schneiden etwas besser ab als die VP in der vom Design her am ehesten vergleichbaren Studie von Krohn/Krohn (2008), die - bezogen auf eine Auswahl aus dem GWS - eine Trefferquote von 57,33\% erzielen (ebd.: 118). Im Vergleich zu den methodisch völlig anders gestalteten Studien von Thomoglou (2004) und Christen (2000) schneiden die Bologneser VP schlechter ab. Bei Thomoglou erreichen die VP in einer mit der Bologneser Gesamtliste vergleichbaren Itemliste eine Trefferquote von 65\% (2004: 84). Die durchschnittliche Korrektheitsquote liegt in der Untersuchung von Christen (2000: 177) quer durch alle Altersgruppen und Lernniveaus bei $78 \%$.

Im Gesamtergebnis erreichen die Feminina mit 69,29\% deutlich das beste Ergebnis, die Maskulina werden zu 58,15\% und die Neutra zu 46,11\% richtig zugewiesen. Dieselbe Reihenfolge ergibt sich bei Christen mit $85 \%$ für Feminina, $74 \%$ für Maskulina und $69 \%$ für Neutra (2000: 177) und auch bei Thomoglou schneiden die Feminina am besten ab. Allerdings erreichen in ihrer Studie Maskulina und Neutra dieselbe Trefferquote und der Abstand zu den Feminina ist weniger ausgeprägt, nämlich 63\% gegenüber 70\% (2004: 85). Das insgesamt schlechte Abschneiden der Neutra ist auch aus dem bilingualen Erstspracherwerb bekannt:

Gender attribution does not seem to be problematic for the children, if one considers nominals containing definite article. Neuter nouns represent an exception to this generalization. [...] With respect to gender, neuter gender remains to be a problem until the age of 5. Most neuter nouns are initially classified as masculine nouns. (Müller 2000: 378, 392)

${ }^{21}$ In Tabelle 1 stehen die Begriffe valide/nicht-valide in Beziehung zu den 11 Regelgruppen. So ist Spirituose ein nicht valides Item für die Regel "Alkoholika sind meist maskulin". Eine Auszählung, die jedes (valide) Item aufgrund der ihm eigenen Zuweisungsregel erfasst, zeigt Tabelle 4. Dort gehört Spirituose zur "Schwa-Regel". 
Anders sind in diesem Zusammenhang die Ergebnisse von Krohn/Krohn (2008: 118), wo die Maskulina mit 59\% am besten abschneiden, gefolgt von den Neutra mit 58\% und den Feminina mit 55\%. Allerdings betrachten die Autoren dieses Ergebnis als nicht signifikant:

Doch liegen die Fehlerquoten der drei Genera [...] u. E. insgesamt so dicht beieinander, dass bereits bei geringfügigen Änderungen der Anzahl und Art von Items die statistische Verteilung ganz anders aussehen kann [...] (ebd.: 118f.)

Nur erwähnt sei hier die Studie von Rogers (1987) mit englischen DaF-Lernenden, in der auch die Feminina die meisten falschen Zuweisungen erhielten. ${ }^{22}$

Nimmt man die validen und die nicht-validen Items zusammen bzw. berücksichtigt nur die validen, so bleibt diese Reihenfolge gleich. Dabei erreichen die Feminina bei den validen Items im GWS eine Trefferquote von 92,82\% und bei B2+ immerhin noch 81,96\%. Bei den nicht-validen Items allerdings schneiden die Feminina bei allen Items und im GWS sogar erheblich schlechter als Maskulina und Neutra ab. Nur bei den (für die jeweilige Regelgruppe) nicht-validen Items B2+ haben die Feminina wieder (mit Abstand) die höchste Trefferquote vor den Neutra und dem einzigen Maskulinum dieser Gruppe. Sieht man sich die femininen Items an, die für ihre jeweilige Regelgruppe nicht-valide sind, so kann man feststellen, dass es sich bei den GWS-Items um wirklich atypische Feminina handelt: Nummer, Ampel, Geburt und Milch (s. u.). Die beiden nicht-validen B2+ Items waren dagegen für die Regel "Alkoholika sind meist maskulinum und Ge-...(-e) als Indiz für Neutrum" jeweils Ausnahmen, aber typische Feminina, weshalb sie - obwohl sie überwiegend unbekannt sein dürften - doch recht hohe Trefferquoten erzielen: Spirituose $(63,7 \%)$ und Gemeinde $(55,53 \%)$. Interessant ist in diesem Zusammenhang auch Geburt, das einen hohen Anteil am schlechten Abschneiden der Feminina im GWS hat. Ihm wurde nur zu 10,57\% das Femininum zugewiesen, obwohl das italienische Äquivalent la nascita feminin ist. Aber in 66,89\% der Fälle hielten es die VP für ein Neutrum. ${ }^{23}$ Aus dem sehr guten Abschneiden der Feminina im Gesamtergebnis und bei den validen Items, aber gerade auch aus dem sehr schlechten Ergebnis bei den nichtvaliden Items kann m. E. geschlossen werden, dass die Lernenden offensichtlich eine klare Vorstellung davon entwickelt haben, wie ein prototypisches Femininum aussieht. Dies bedeutet auch, dass Lernende grundsätzlich - wie auch immer beschaffene - Regeln der Genuszuweisung kennen und anwenden.

In diese Richtung weist auch das insgesamt schlechte Abschneiden der Neutra, auch wenn die Tatsache, dass von 14 neutralen Items 8 nicht-valide Beispiele für die jeweilige Regelgruppe sind, sicher auch eine Rolle spielt. Damit haben die Neutra den größten Anteil an nichtvaliden Items überhaupt gegenüber 2 maskulinen und 6 femininen Ausnahmen:

\footnotetext{
22 Das schlechte Abschneiden der Feminina wurde bei englischen und schwedischen, das gute bei französischen, italienischen und griechischen Deutschlernenden festgestellt. Interessant wäre hier zu untersuchen, ob dies auf den Einfluss der Muttersprache zurückgeführt werden kann.

23 Bei der Regel "Mit $\mathrm{Ge}$-...(-e) gebildete Kollektiva sind neutrum" ist zu bedenken, dass für den Lernenden das Merkmal "Kollektivum" ggf. hinter das formale Merkmal zurücktreten kann.
} 


\begin{tabular}{|l|l|c|c|c|c|}
\hline & Item & $\mathbf{m}$ & $\mathbf{f}$ & $\mathbf{n}$ & $\varnothing$ \\
\hline 1. & Kind & 6,55 & 0,00 & 92,94 & 0,52 \\
\hline 2. & Bier & 4,24 & 4,24 & 91,01 & 0,52 \\
\hline 3. & Hören & 3,69 & 19,98 & 71,59 & 4,74 \\
\hline 4. & Geschirr & 22,68 & 3,09 & 61,86 & 12,37 \\
\hline 5. & *Gebäck & 16,49 & 11,34 & 60,82 & 11,34 \\
\hline 6. & Schreiben & 14,43 & 18,56 & 59,79 & 7,22 \\
\hline 7. & *Fach & 36,41 & 13,28 & 44,99 & 5,32 \\
\hline 8. & Gemüse & 3,16 & 56,82 & 36,33 & 3,69 \\
\hline 9. & Kissen & 13,68 & 37,61 & 31,77 & 16,94 \\
\hline 10 & *Silber & 54,38 & 8,48 & 24,44 & 12,71 \\
\hline 11. & *Züchten & 13,60 & 39,96 & 21,85 & 24,59 \\
\hline 12. & *Küken & 10,06 & 53,84 & 19,55 & 16,56 \\
\hline 13. & *Mündel & 30,57 & 31,20 & 15,40 & 22,84 \\
\hline 14. & *Rudel & 55,55 & 11,60 & 13,20 & 19,65 \\
\hline
\end{tabular}

Tab. 2: Zuweisungen zu den Neutra ${ }^{24}$

Wenn man jedoch bedenkt, dass bei einer generellen Trefferquote von 46,11\% selbst bei den validen Items aus dem GWS für die Neutra nur in 57,39\% der Fälle eine korrekte Zuweisung erfolgt, scheinen die Ausnahmen nicht das alleinige Problem darzustellen. Dies zeigt sich auch daran, dass die nicht-validen GWS-Items Kind und Bier eine Trefferquote von jeweils über $90 \%$ erzielen. Allerdings scheint in unserer Untersuchung - anders als in der Untersuchung von Müller zum bilingualen Erstspracherwerb - bei falschen Zuweisungen nicht generell das Maskulinum statt des Neutrums gewählt zu werden, sondern regelbasierte Überlegungen eine Rolle zu spielen. Auffallend ist dies bei Gemüse, das in über 56\% der Fälle als Femininum klassifiziert wird. Das Maskulinum scheint nur dann eine wirkliche Option zu sein, wenn es keine Indizien für Femininum gibt: Einsilber (Fach), -er (Silber), -el (Rudel). Die hohe bis sehr hohe Zuweisung des Femininum bei den Substantiven auf -en könnte dagegen bedeuten, dass hier nicht unbedingt ein Genus zugewiesen, sondern die als nicht analysierter Teil der Pluralform die ...-en gewählt wird (dies geht aus den Antworten zu Aufgabe 2 hervor). Nur bei Mündel erreicht die Zuweisung von Maskulinum und Femininum fast gleiche Werte.

Wie oben bereits erwähnt, liegt das Ergebnis für den GWS deutlich unter unseren Erwartungen. Schaut man sich aber die Ergebnisse zu den einzelnen Items an, ergibt sich ein differenzierteres Bild. Im GWS liegt der Range zwischen 10,81\% und 100\%. 14 Items haben eine Trefferquote von $75 \%$ und höher, 6 Items liegen zwischen $60 \%$ und $72 \%$ und nur 10 Items erreichen weniger als $45 \%$. Nimmt man nur die 20 besten Items, so ergibt sich eine Trefferquote von immerhin $83,1 \%$, berücksichtigt man nur die validen Items, wird eine Quote von 72,37\% erreicht. Die Abweichung vom erwarteten Ergebnis im GWS ergibt sich also aus einer Gruppe von 10 Items, die hier näher analysiert werden sollen:

\footnotetext{
${ }^{24}$ Items aus B2+ sind mit Asterisk, für ihre jeweilige Regelgruppe nicht-valide Items rot markiert. Das Symbol $\varnothing$ steht für "keine Zuweisung".
} 


\begin{tabular}{|l|l|c|c|c|c|c|}
\hline & \multirow{2}{*}{ Item } & korrekte & \multicolumn{5}{|l|}{ nicht korrekte Zuweisung } \\
\cline { 5 - 7 } & & Zuweisung & m & f & n & $\varnothing$ \\
\hline 21. & Schlüssel & 43,09 & & 32,98 & 16,49 & 7,45 \\
\hline 22. & Durst & 42,23 & & 19,59 & 21,65 & 16,49 \\
\hline 23. & Schnaps & 36,46 & & 18,75 & 26,56 & 18,23 \\
\hline 24. & Gemüse & 36,33 & 3,16 & 56,82 & & 3,69 \\
\hline 25. & Nummer & 33,88 & 33,43 & & 29,14 & 3,56 \\
\hline 26. & Regen & 32,99 & & 12,37 & 49,48 & 5,15 \\
\hline 27. & Kissen & 31,77 & 13,68 & 37,61 & & 16,94 \\
\hline 28. & Ampel & 24,74 & 53,00 & & 7,39 & 14,88 \\
\hline 29. & Milch & 24,60 & 25,17 & & 47,76 & 2,48 \\
\hline 30. & Geburt & 10,57 & 18,00 & & 66,89 & 4,54 \\
\hline
\end{tabular}

Tab. 3: Die 10 schlechtesten Items im GWS

Fünf der 10 Items mit der niedrigsten Trefferquote sind idiosynkratische Substantive. Aber auch die validen Items gehören zu Regelgruppen mit einer eher eingeschränkten Validität: -en und -el sowie Einsilber. Darüber hinaus haben die Substantive weitere Eigenschaften, die den Genuserwerb offensichtlich erschweren. Ein Teil der Items ist zwar Teil des GWS, meiner langjährigen Lehrerfahrung nach aber in der Unterrichtssituation wenig frequent, sei es, weil sie kaum kommunikativen Bedürfnissen von etwa 20-jährigen italienischen Deutschlernenden entsprechen wie Kissen, sei es, weil zwar über die Sache gesprochen wird, aber nicht unbedingt nominal wie im Fall von Regen, wo man in der face-to-face-Kommunikation doch eher auf die verbale Form es regnet zurückgreift. Ähnliches lässt sich zu Geburt sagen, das zwar als Basislexem wichtig ist, aber im Gebrauch eher in Zusammensetzungen wie Geburtstag oder -ort vorkommt, bei denen das Genus durch das jeweilige Grundwort bestimmt wird. Dazu kommt, dass auch hier die Geburt in der Regel mithilfe des verbalen Ausdrucks geboren sein/werden zur Sprache gebracht wird. Auch Schnaps gehört offensichtlich nicht zum geläufigen Wortschatz junger Leute. ${ }^{25}$ Andere Items werden dagegen meist ohne Artikel verwendet wie Durst oder Milch. Interessant ist das Beispiel Schlïssel. Obwohl im Fragebogen ausdrücklich darauf hingewiesen wird, dass alle Items im Nominativ Singular stehen, wird die Form überraschend oft als Pluralform interpretiert (dies wird aus den Antworten in Aufgabe 2 ersichtlich).

Allerdings scheinen sich die Lernenden aber selbst in diesen Fällen nicht einfach aufs Raten zu verlegen. Ein erster allgemeiner Hinweis darauf ist der teilweise doch recht hohe Prozentsatz für "keine Antwort". Eindeutig im "Ratebereich" liegt nur Nummer. An diesem nichtvaliden Item wird aber auch deutlich, was sich auch zu den meisten anderen Items dieser Gruppe sagen lässt, nämlich dass auch die Übertragung des Genus aus der Muttersprache offensichtlich keine generelle Zuweisungsstrategie darstellt. ${ }^{26}$ Obwohl das Genus von Nummer auch unserer Erfahrung nach den Lernenden hartnäckige Probleme bereitet, weisen sie nicht überwiegend den dem italienischen Äquivalent il numero entsprechenden maskulinen Artikel zu. Bei nur zwei Items könnte es sich um Transfer handeln. So wird Ampel in mehr als 50\% der Fälle der dem italienischen Äquivalent il semaforo entsprechende maskuline Artikel zugewiesen, Gemüse sogar in mehr als $56 \%$ der dem italienischen la verdura entsprechende feminine Artikel. Bei letzterem könnte allerdings auch die "Schwa-Regel" eine eventuelle Transferstrategie verstärken. Bei Ampel könnte neben dem Einfluss der Muttersprache auch

\footnotetext{
${ }^{25}$ Wir hatten - fälschlicherweise - angenommen, Schnaps sei ähnlich bekannt als typisches Getränk wie Bier. ${ }^{26}$ Vgl. dazu auch Christen (2000: 182).
} 
die Tatsache eine Rolle spielen, dass das Lexem oft in Phrasen wie an der Ampel verwendet wird. Gegen eine generelle Anwendung von Transfer sprechen auch die Ergebnisse von Milch, das - innerhalb des GWS - die zweitniedrigste Trefferquote überhaupt erreicht. Erwartet hätten wir hier eine klare Tendenz zum Maskulinum aufgrund des italienischen il latte. Dagegen wird dem Substantiv in nahezu 50\% der Fälle Neutrum zugewiesen. Gleiches gilt für Regen, wo sogar nur zu 12,37\% das aus dem Italienischen erwartbare Femininum (la pioggia) zugewiesen wird. In diesem Zusammenhang sei noch einmal auf Geburt hingewiesen, das, obwohl aus dem GWS und obwohl im Italienischen Femininum (la nascita), das zweitschlechteste Ergebnis überhaupt erzielt, nämlich 10,57\%. Stattdessen scheint die "Ge-(e)Regel" zur Anwendung zu kommen, denn in 66,89\% der Fälle wird hier das Neutrum zugewiesen. Hier kann außerdem die Hypothese formuliert werden, dass Lernende dann auf die "Ge-(e)-Regel" zurückgreifen, wenn es keine Anhaltspunkte für andere Zuweisungskriterien gibt wie bei Geschirr, Gebäck und Geburt, die ähnlich hohe Werte bei der Zuweisung des Neutrums erreichen.

Der detaillierte Blick auf die Ergebnisse im Bereich GWS scheint in folgende Richtung zu weisen: Obwohl die explizite Vermittlung von Genusregeln in Unterricht und Lehr-/ Lernmaterialien eine eher untergeordnete Rolle spielt, kennen die VP eine Reihe von Regeln, die sie bei der Genuszuweisung anwenden. Da aber auch die idiosynkratischen Items Kind und Bier Trefferquoten von über 93\% erzielen, spielt auch die Frequenz bzw. der Bekanntheitsgrad eine nicht unbedeutende Rolle.

Die Trefferquoten der Items auf Niveau B2+ bestätigen, dass bei der Genuszuweisung offensichtlich Regelwissen zum Einsatz kommt. Immerhin zu 8 der weitgehend wohl unbekannten 19 Items wird in mehr als $60 \%$ der Fälle das Genus richtig zugewiesen mit Spitzenwerten für Richterin $(95,36 \%)$, Spitzer $(87,58 \%)$ und Verlobung $(86,06)$. Fast einen schlagenden Beweis in dieser Richtung könnte man in der Zuweisung für das sicher unbekannte und in seiner Regelgruppe nicht-valide Funke sehen, dem in 63,38\% der Fälle Femininum zugewiesen wird, also vergleichbar oft wie Waise $(64,43 \%)$ und Spirituose $(63,70 \%)$.

Für die 11 Regelgruppen werden folgende Trefferquoten erreicht:

\begin{tabular}{|l|c|c|c|c|c|c|c|}
\hline \multicolumn{1}{|c|}{ Regel } & Validität (\%) & & Alle & & GWS & & B2+ \\
\hline -in & 100 & & 98,20 & & 99,62 & & 95,36 \\
\hline Sexusregel & 86,1 & & 92,25 & & 93,44 & & 88,66 \\
\hline -ung & 100 & & 89,17 & & 90,72 & & 86,08 \\
\hline -er & 65 & & 85,81 & & 86,86 & & 84,77 \\
\hline$-e$ & 90,5 & & 72,20 & & 88,66 & & 61,22 \\
\hline Einsilber & 51,8 & & 56,86 & & 56,43 & & 57,73 \\
\hline Ge- & $?$ & & 53,00 & & 49,10 & & 60,82 \\
\hline $\begin{array}{l}\text { substantivierte } \\
\text { Verben }\end{array}$ & 100 & & 51,08 & & 65,69 & & 21,85 \\
\hline -el & 60,5 & & 50,00 & & 55,57 & & 38,86 \\
\hline -en & 72,1 & & 45,61 & & 56,19 & & 24,46 \\
\hline Semantische Regel & $?$ & & 38,78 & & 54,31 & & 7,73 \\
\hline
\end{tabular}

Tab. 4: Trefferquoten (\%) bei den validen Items nach Regelgruppen

In den Durchschnittswerten von Tabelle 4 sind jeweils alle Items enthalten, denen das Genus aufgrund einer Regel zugewiesen werden kann. So ist z. B. Junge im Fragebogendesign eine Ausnahme von der "-e-Regel", gehört aber grundsätzlich in die Gruppe "Sexusregel" und wird 
deshalb in der obigen Tabelle in dieser Gruppe gezählt. Andere Items wie Schwager oder Studentin können zwei verschiedenen Regeln zugeordnet werden. Da in Aufgabe 2 bei den Items, deren Genus sowohl aufgrund der Sexusregel als auch aufgrund morphologischer Merkmale bestimmt werden kann, beide Regeln (in einigen Fällen von derselben VP) genannt werden, zählen sie in beiden Gruppen.

Insgesamt spiegelt Tabelle 4 die Ergebnisse wider, die sich bisher aus der Detailanalyse ergeben haben. Dass sich die Trefferquoten in keinem Fall um den Zufallswert herum einpendeln, kann sicher so interpretiert werden, dass die Lernenden auch bei der oft als (überwiegend) regellos vermittelten Genuszuordnung Regeln entwickeln und anwenden. Der Grad an Sicherheit bei der Genuszuweisung scheint dagegen ein Zusammenspiel ${ }^{27}$ verschiedener Faktoren zu sein. So zeichnen sich die Items auf -in, die am besten abschneiden und bezogen auf alle Items bzw. auf den GWS eine Trefferquote von nahezu $100 \%$ erzielen, durch folgende Merkmale aus:

a) Die Items aus dem GWS Lehrerin und Studentin haben eine hohe Relevanz sowohl für die Lernenden als auch für die Situation Unterricht.

b) Das Genus kann aufgrund von 2 verschiedenen Regeln zugewiesen werden, nämlich der Sexusregel und einer morphologischen Regel.

c) Das Suffix -in hat eine klar erkennbare Funktion, nämlich die der Kennzeichnung des natürlichen Geschlechts.

d) Die Regel hat eine Validität von 100\%.

e) Das Suffix ist so prominent, dass es auch im Falle von weniger frequenten bzw. unbekannten Substantiven in seiner genuszuweisenden Funktion erkannt wird.

Mit der hohen Trefferquote bei der Sexusregel, wo selbst auf B2+ über 88\% der Zuweisungen korrekt sind, bestätigen auch die Bologneser Ergebnisse die prominente Rolle, die dieses Zuweisungskriterium im (nicht muttersprachlichen) Spracherwerb offensichtlich spielt. So berichtet Wegener in Bezug auf den Zweitspracherwerb von Kindern, dass es sich bei der Sexusregel um die erste von den VP entdeckte Genusregel handelt (1995: 14). Auch bei der "-ung-Regel" werden noch gute Werte erreicht, aber trotz der 100\%-Validität der Regel liegt die Trefferquote "nur" bei etwa $90 \%$ bzw. darunter. So treffen bei der "-ung-Regel" auch nur die Faktoren a), d) und e) zu. Die Bedeutung des Bekanntheitsgrades in Verbindung mit der Prominenz des Merkmals wird m. E. durch die Ergebnisse in Bezug auf die "-e-Regel" unterstrichen. Obwohl die Regel in Aufgabe 2 sehr oft genannt wird - vielen VP also bekannt ist -, erreichen die richtigen Zuweisungen für alle Items nur 72,20\%, ein Wert, der im Bereich GWS auf 88,66\% ansteigt, für B2+ aber auf $61,62 \%$ abfällt. Somit erreicht die sehr valide "-eRegel" für Items aus B2+ in etwa dieselben Werte wie die sehr viel weniger valide "Ge-" und die "Einsilber-Regel".

Dass die Lernenden zwar ein bestimmtes Regelwissen besitzen, dies aber nicht konsequent abrufen und anwenden (können), zeigt auch ein Blick in die frei formulierten Zuweisungskriterien in Aufgabe 2. Auch wenn die statistische Auswertung noch nicht abgeschlossen ist, so zeigt sich ein großes Unsicherheitspotential allein darin, dass die VP 21 verschiedene Regeln nennen, darunter viele nicht existierende - aber nicht unbedingt idiosynkratische - Regeln wie "Substantive auf - $c k$ sind Neutrum" und "Substantive auf $-s$ sind Neutrum". Dazu kommen sechs semantische Regeln, darunter "Gemüse und Pflanzen sind Neutrum" und "Abstrakta sind Neutrum". Schließlich acht Regeln zum Bereich Wortbildung und Fremdwörter ("Fremdwörter sind Neutrum"), aber auch Überlegungen wie "Sprachgefühl", "Eselsbrücke" und "Zufallsprinzip". Ein noch stärkerer Hinweis auf die relative "Flüchtigkeit" des Regelwis-

\footnotetext{
${ }^{27}$ Die Betonung liegt hier auf Zusammenspiel, weshalb die folgende Aufzählung nicht als Rangfolge zu verstehen ist.
} 
sens als in diesem Sammelsurium an (auch) richtigen und nur im Kopf der VP existierenden Regeln ist die auffallend häufige Beobachtung, dass z. B. Spitzer (Item 10 in Aufgabe 2 der B-Version) das maskuline Genus aufgrund der explizit genannten "-er-Regel" zugewiesen wird, bei Item 11 Schwager aber nicht.

\section{$5 \quad$ Ausblick}

Insgesamt hat die Untersuchung unsere Ausgangshypothesen nicht bestätigt. Die Trefferquoten im GWS sind eindeutig niedriger als unsere Erwartungen, gleichzeitig erfolgt die Genuszuweisung offensichtlich häufiger aufgrund von Regeln und auch die Teilauswertung von Aufgabe 2 zeigt, dass die VP mehr Regeln kennen und verbalisieren können als erwartet. Der Bekanntheitsgrad (aufgrund von Gebrauchshäufigkeit und/oder kommunikativen Bedürfnissen) spielt zwar offensichtlich eine große Rolle, was auch durch die hohen Trefferquoten bei einigen idiosynkratischen Substantiven wie Kind und Bier unterstrichen wird. Doch sollte dies keine Begründung für den immer noch geringen Stellenwert darstellen, der dem Bereich Genus in den Lehr-/Lernmaterialien eingeräumt wird. Denn die Genuszuordnung ist im Deutschen nicht nur arbiträr, es gibt durchaus eine Reihe von Regeln, derer sich offensichtlich auch Lernende bedienen. Oder muss hier vermutet werden, dass an der Schnittstelle zwischen linguistischer und didaktischer Grammatik statt einer begründeten Inhaltsauswahl, wo nach Zimmermann "die linguistischen Daten unter dem Stichwort Lerninhalte ins Spiel" (2003: 407; Hervorhebung im Original) kommen sollten, vielmehr das Prinzip Alles oder Nichts zum Tragen kommt, wobei anscheinend die schiere Fülle dann für Letzteres den Ausschlag gibt?

Im Rahmen dieses Beitrags muss offen bleiben, auf welche Weise sich Genusregeln in den (mentalen) Lernergrammatiken ausbilden. Trotzdem gibt die Untersuchung m. E. wertvolle Hinweise darauf, wie Unterricht und Lehr-/Lernmaterialien die Herausbildung und Anwendung des Regelapparats unterstützen können. Wie in anderen Bereichen sollte auch hier im Sinne des language awareness-Konzeptes bei der Bewusstmachung von (hoch-validen) systemlinguistischen Regeln angesetzt werden. Die Umsetzung der Bewusstheit in Sprachpraxis muss dann aber durch ein systematisches Strategietraining gefördert werden, in dem die Lernenden in der Anwendung von (genus-bezogenen) Lern- und Gebrauchsstrategien unterstützt werden. Denn gerade das Auseinanderklaffen von bei den VP vorhandenem verbalisierbarem Regelwissen einerseits und richtiger Zuweisung andererseits wie im Fall der absoluten "-ungRegel" bzw. der hoch-validen "-e-Regel" zeigt, dass es an Bewusstsein und prozeduralem Wissen darüber fehlt, dass Regelwissen strategisch eingesetzt werden kann (was für Strategien ja im Allgemeinen gilt).

\section{Literatur}

Bittner, Dagmar (2000): "Gender classification and the inflectional system of German nouns". In: Unterbeck, Barbara et al. (eds.): Gender in Grammar and Cognition. Berlin/New York, Mouton de Gruyter: 1-23.

Christen, Helen (2000): "'Der Brot, die Mädchen, das Führerschein'. Der Erwerb der deutschen Genera". In: Diehl, Erika et al. (2000): Grammatikunterricht: Alles für der Katz? Untersuchungen zum Zweitspracherwerb Deutsch. Tübingen, Niemeyer: 167-187.

Corbett, Greville G. (1991): Gender. Cambridge: Cambridge University Press.

Diehl, Erika et al. (2000): Grammatikunterricht: Alles für der Katz? Untersuchungen zum Zweitspracherwerb Deutsch. Tübingen: Niemeyer.

Glabionat, Manuela et al. (2002): Profile deutsch. Gemeinsamer europäischer Referenzrahmen. Lernzielbestimmungen, Kannbeschreibungen, Kommunikative Mittel Niveau A1, A2, $B 1, B 2$. Berlin etc.: Langenscheidt. 
Helbig, Gerhard/Buscha, Joachim (2001): Deutsche Grammatik. Ein Handbuch für den Ausländerunterricht. Berlin etc.: Langenscheidt.

Hockett, Charles F. (1958): A course in modern linguistics. New York: Macmillan.

Ivancic, Barbara (2003): Deutsche Sprache, schwere Sprache - Ma le grammatiche ci aiutano? Considerazioni sulle grammatiche didattiche del tedesco. Trieste: EUT.

Köpcke, Klaus-Michael/Zubin, David A. (1983): "Die kognitive Organisation der Genuszuweisung zu den einsilbigen Nomen der deutschen Gegenwartssprache". Zeitschrift für germanistische Linguistik 11: 166-182.

Köpcke, Klaus-Michael/Zubin, David A. (1984): "Sechs Prinzipien für die Genuszuweisung im Deutschen: Ein Beitrag zur natürlichen Klassifikation". Linguistische Berichte 93: 26-50.

Köpcke, Klaus-Michael/Zubin, David A. (1996): "Prinzipien für die Genuszuweisung im Deutschen". In: Lang, Ewald/Zifonun, Gisela (eds.): Deutsch - typologisch. Berlin/New York, de Gruyter: 473-491.

Krohn, Dieter/Krohn, Karin (2008): Der, das, die - oder wie? Studien zum Genuserwerb schwedischer Deutschlerner. Frankfurt am Main etc.: Lang.

Leiss, Elisabeth (1994): "Genus und Sexus. Kritische Anmerkungen zur Sexualisierung von Grammatik". Linguistische Berichte 152: 281-300.

Leiss, Elisabeth (2000): "Gender in Old High German". In: Unterbeck, Barbara et al. (eds.): Gender in Grammar and Cognition. Berlin/New York, Mouton de Gruyter: 237-258.

Menzel, Barbara (2004): Genuszuweisung im DaF-Erwerb. Psycholinguistische Prozesse und didaktische Implikationen. Berlin: Weißensee.

Müller, Natascha (2000): "Gender and number in acquisition". In: Unterbeck, Barbara et al. (eds.): Gender in Grammar and Cognition. Berlin/New York, Mouton de Gruyter: 351-399.

Rogers, Margaret (1987): "Learners Difficulties with Grammatical Gender in german as a Foreign Language". Applied Linguistics 8: 48-74.

Sieburg, Heinz (ed.) (1997): Sprache - Genus/Sexus. Frankfurt am Main etc.: Lang.

Spillner, Bernd (2003): "Angewandte Linguistik". In: Bausch, Karl-Richard/Christ, Herbert/Krumm, Hans-Jürgen (eds.): Handbuch Fremdsprachenunterricht. Tübingen, A. Francke: $31-37$.

Thomoglou, Polichronia (2004): Genuserwerb bei griechischen Lernern des Deutschen. Frankfurt am Main etc.: Lang.

Unterbeck, Barbara (2000): "Gender: New light on an old category". In: Unterbeck, Barbara et al. (eds.): Gender in Grammar and Cognition. Berlin/New York, Mouton de Gruyter: XV-XLVI.

Vogel, Petra M. (2000): "Nominal abstracts and gender in Modern German: A 'quantitative' approach towards the function of gender". In: Unterbeck, Barbara et al. (eds.): Gender in Grammar and Cognition. Berlin/New York, Mouton de Gruyter: 461-493.

Weber, Doris (2000): "Gender - a nominal category without any function?" In: Unterbeck, Barbara et al. (eds.): Gender in Grammar and Cognition. Berlin/New York, Mouton de Gruyter: 495-509.

Wegener, Heide (1995): "Das Genus im DaZ-Erwerb". In: Handwerker, Brigitte (ed.): Fremde Sprache Deutsch: grammatische Beschreibung - Erwerbsverläufe - Lehrmethodik. Tübingen, Narr: 1-24.

Wegener, Heide (2000): "German gender in children's second language acquisition". In: Unterbeck, Barbara et al. (eds.): Gender in Grammar and Cognition. Berlin/New York, Mouton de Gruyter: 511-544.

Zimmermann, Günther (2003): "Grammatiken". In: Bausch, Karl-Richard/Christ, Herbert/Krumm, Hans-Jürgen (eds.): Handbuch Fremdsprachenunterricht. Tübingen, A. Francke: 406-409. 


\section{Anhang}

Itemliste 28 nach Regelgruppen geordnet mit den auf alle Aufgaben bezogenen Durchschnittswerten $(\%)$ :

\begin{tabular}{|c|c|c|c|c|c|}
\hline & Item & der & die & das & $\varnothing$ \\
\hline 1. & Tochter & 4,12 & 91,75 & 3,61 & 0,52 \\
\hline 2. & Sohn & 93,81 & $\mathbf{0 , 0 0}$ & 3,09 & 3,09 \\
\hline 3. & Schwager & 81,96 & 4,64 & 2,58 & 10,82 \\
\hline 4. & Kind & 6,55 & $\mathbf{0 , 0 0}$ & 92,94 & $\mathbf{0 , 5 2}$ \\
\hline 5. & Mündel & $\mathbf{3 0 , 5 7}$ & 31,20 & 15,40 & 22,84 \\
\hline 6. & Schnaps & 36,46 & 18,75 & 26,56 & 18,23 \\
\hline 7. & Wein & 72,16 & 6,19 & 18,56 & 3,09 \\
\hline 8. & Wodka & 7,73 & 38,14 & 44,33 & 9,79 \\
\hline 9. & Bier & 4,24 & 4,24 & 91,01 & 0,52 \\
\hline 10. & Spirituose & 7,02 & 63,70 & 11,60 & 17,69 \\
\hline 11. & Wohnung & 4,12 & 92,78 & 2,06 & 1,03 \\
\hline 12. & Rechnung & 7,22 & 88,66 & 2,06 & 2,06 \\
\hline 13. & Verlobung & 4,64 & 86,08 & 3,09 & 6,19 \\
\hline 14. & Hören & 3,69 & 19,98 & 71,59 & 4,74 \\
\hline 15. & Schreiben & 14,43 & 18,56 & 59,79 & 7,22 \\
\hline 16. & Züchten & 13,60 & 39,96 & 21,85 & 24,59 \\
\hline 17. & Lehrerin & 0,78 & 99,23 & 0,00 & $\mathbf{0 , 0 0}$ \\
\hline 18. & Studentin & 0,00 & 100,00 & 0,00 & 0,00 \\
\hline 19. & Richterin & 0,00 & 95,36 & 0,52 & 4,12 \\
\hline 20. & Reise & 5,67 & 92,78 & 0,52 & 1,03 \\
\hline 21. & Blume & 9,28 & 84,54 & 3,09 & 3,09 \\
\hline 22. & Waise & 15,46 & 64,43 & 4,64 & 15,46 \\
\hline 23. & Junge & 78,94 & 18,44 & 2,12 & 0,52 \\
\hline 24. & Funke & 15,06 & 63,38 & $\mathbf{5 , 5 2}$ & 16,04 \\
\hline 25. & Fernseher & 76,80 & 5,15 & 16,49 & 1,55 \\
\hline 26. & Lehrer & 96,91 & 1,03 & 2,06 & 0,00 \\
\hline 27. & Spitzer & 87,58 & 2,07 & 2,59 & 7,76 \\
\hline 28. & Nummer & 33,43 & 33,88 & 29,14 & 3,56 \\
\hline 29. & Silber & 54,38 & 8,48 & 24,44 & 12,71 \\
\hline 30. & Schlüssel & 43,09 & 32,98 & 16,49 & 7,45 \\
\hline 31. & Apfel & 68,04 & 23,71 & 5,15 & 3,09 \\
\hline 32. & Beutel & 38,86 & 9,32 & 30,58 & 21,24 \\
\hline 33. & Ampel & 53,00 & 24,74 & 7,39 & 14,88 \\
\hline 34. & Rudel & 55,55 & 11,60 & 13,20 & 19,65 \\
\hline 35. & Gemüse & 3,16 & 56,82 & 36,33 & 3,69 \\
\hline 36. & Geschirr & 22,68 & 3,09 & 61,86 & 12,37 \\
\hline 37. & Gebäck & 16,49 & 11,34 & 60,82 & 11,34 \\
\hline 38. & Geburt & 18,00 & 10,57 & 66,89 & 4,54 \\
\hline 39. & Gemeinde & 3,10 & 55,53 & 21,24 & 20,15 \\
\hline 40. & Garten & 79,38 & 6,70 & 11,34 & 2,58 \\
\hline 41. & Regen & 32,99 & 12,37 & 49,48 & 5,15 \\
\hline 42. & Nacken & 24,46 & 32,12 & 17,84 & 25,58 \\
\hline 43. & Kissen & 13,68 & 37,61 & 31,77 & 16,94 \\
\hline 44. & Küken & 10,06 & 53,84 & 19,55 & 16,56 \\
\hline 45. & Brief & 70,62 & 5,15 & 22,68 & 1,55 \\
\hline 46. & Durst & 42,23 & 19,59 & 21,65 & 16,49 \\
\hline 47. & Sund & 57,73 & 10,31 & 13,40 & 18,56 \\
\hline 48. & Milch & 25,17 & 24,60 & 47,76 & 2,48 \\
\hline 49. & Fach & 36,41 & 13,28 & 44,99 & 5,32 \\
\hline
\end{tabular}

${ }^{28}$ Items aus dem Bereich B2+ sind kursiv, für die jeweilige Regelgruppe nicht-valide Items rot markiert; das Symbol $\varnothing$ steht für "keine Angaben". 